\title{
Kinetics of Network Formation and Heterogeneous Dynamics of an Egg White Gel Revealed by Coherent X-Ray Scattering
}

\author{
Nafisa Begam $\odot,{ }^{1, *}$ Anastasia Ragulskaya, ${ }^{1}$ Anita Girelli®,${ }^{1}$ Hendrik Rahmann, ${ }^{2}$ Sivasurender Chandran $\odot,{ }^{3}$ \\ Fabian Westermeier $\odot{ }^{4}$ Mario Reiser, ${ }^{2,5}$ Michael Sprung, ${ }^{4}$ Fajun Zhang $\odot,{ }^{1, \dagger}$ Christian Gutt๑, ${ }^{2, \$}$ and Frank Schreiber $\oplus^{1, \S}$ \\ ${ }^{1}$ Institut für Angewandte Physik, Universität Tübingen, 72076 Tübingen, Germany \\ ${ }^{2}$ Department Physik, Universität Siegen, 57072 Siegen, Germany \\ ${ }^{3}$ Department of Physics, Indian Institute of Technology Kanpur, Kanpur, Uttar Pradesh 208016, India \\ ${ }^{4}$ Deutsches Elektronen-Synchrotron DESY, Notkestrasse 85, 22607 Hamburg, Germany \\ ${ }^{5}$ European X-ray Free-Electron Laser GmbH, Holzkoppel 4, 22869 Schenefeld, Germany
}

(Received 23 July 2020; revised 12 November 2020; accepted 11 January 2021; published 2 March 2021)

\begin{abstract}
The kinetics of heat-induced gelation and the microscopic dynamics of a hen egg white gel are probed using x-ray photon correlation spectroscopy along with ultrasmall-angle x-ray scattering. The kinetics of structural growth reveals a reaction-limited aggregation process with a gel fractal dimension of $\approx 2$ and an average network mesh size of ca. $400 \mathrm{~nm}$. The dynamics probed at these length scales reveals an exponential growth of the characteristic relaxation times followed by an intriguing steady state in combination with a compressed exponential correlation function and a temporal heterogeneity. The degree of heterogeneity increases with decreasing length scale. We discuss our results in the broader context of experiments and models describing attractive colloidal gels.
\end{abstract}

DOI: 10.1103/PhysRevLett.126.098001

Egg white, apart from being a major protein source in our daily diet, is one of the most versatile products in the food industry due to its multifunctional properties such as foaming, emulsifying, and gelling [1-5]. Gelation of proteins [6], especially as a result of heat-induced denaturation, leads to a three-dimensional network structure through the formation of disulfide cross-links and hydrogen bonds $[1,7,8]$. While various experiments mainly focused on the understanding of structural evolution at the molecular level [1,9], the corresponding evolution of the microscopic dynamics in a heat-treated protein gel has not been fully explored yet.

So far, the studies of the dynamic properties of thermal gels of proteins have only contributed to the understanding of internal or short-time self-diffusion processes in protein systems occurring at nanosecond and subnanosecond timescales $[10,11]$. However, the process of protein gelation involves rather a hierarchy of length and timescales. This requires the understanding of the structure and dynamics of protein gels on length scales ranging from single proteins up to the network mesh size (hundreds of nanometers to micrometers), and timescales of milliseconds to hundreds of seconds. This constitutes a considerable challenge both for simulations and experiments with the need to capture especially nonequilibrium phenomena such as aging and dynamical heterogeneity.

Applying state-of-the-art low-dose x-ray photon correlation spectroscopy (XPCS) along with ultrasmall-angle $\mathrm{x}$-ray scattering (USAXS), we follow here the simultaneous evolution of the microscopic dynamics on length scales of the network mesh size and the structural evolution corresponding to the gelation kinetics of hen egg white. We show that, under the chosen conditions, the kinetic evolution of the gel network can be remarkably well separated from the dynamics. We find that the gel exhibits stress-activated dynamics with an exponential rise of the relaxation time (aging) and a subsequent steady-state ballistic motion displaying significant temporal heterogeneity. Our experiments show that the dynamical aging sets in at timescales well beyond the formation time of the network structure. We identify the stress-driven dynamics as dynamical rupture events which do not change the structure of the gel. The spatial extension of these decorrelation events decreases from $100 \mathrm{~nm}$ to a few $\mathrm{nm}$ upon aging accompanied by a lowering of the degree of dynamical heterogeneity.

The hen egg sample was purchased from a supermarket. The egg white was extracted in the laboratory, filled in quartz capillaries with a diameter of $1.5 \mathrm{~mm}$, sealed with parafilm, and mounted on a sample stage equipped with a temperature-controlled heating stage (Linkam Scientific Instruments Ltd., UK) to perform XPCS experiments in USAXS geometry $[12,13]$ at the beam line P10 of PETRA III (DESY, Hamburg, Germany, with $\lambda=1.54 \AA$ ) [14]. Applying a large beam size of $100 \times 100 \mu \mathrm{m}^{2}$ allowed us to perform the experiment at $\mathrm{x}$-ray doses below the damage threshold. We recorded a series of time-resolved scattering patterns (with a temporal resolution of $40 \mathrm{~ms}$ ) using an area detector, Eiger X $4 \mathrm{M}\left(75 \times 75 \mu \mathrm{m}^{2}\right.$ pixel size $)$ mounted $21.3 \mathrm{~m}$ downstream of the sample (details in the Supplemental Material [15]). 


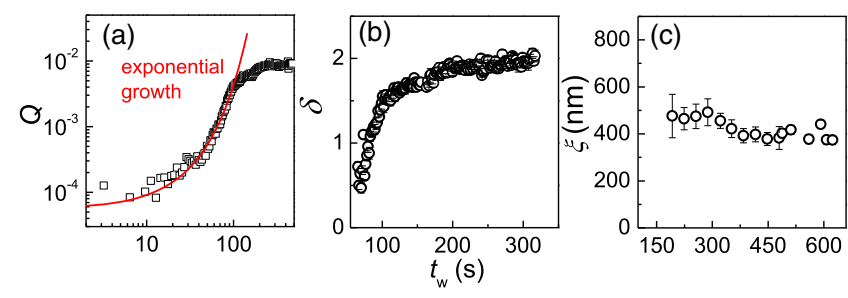

FIG. 1. (a) Invariant as a function of $t_{w}$ calculated over the $q$ range of $0.003-0.1 \mathrm{~nm}^{-1}$, showing an exponential growth (red solid line) of aggregates, (b) fractal dimension $\delta$ as a function of $t_{w}$ obtained at the low $q$ region of the USAXS profiles, and (c) mesh size, as extracted from Ornstein-Zernike plot, is shown as a function of $t_{w}$.

We first investigate the growth kinetics of the gel aggregates by calculating the scattering invariant $Q$ [16] from the intensity profiles $I(q)$ (see Fig. S2 in the Supplemental Material [15]), which gives an estimation of the flocculation parameter [17]. An exponential growth of $Q$ as a function of the waiting time $t_{w}$ is observed in Fig. 1(a) suggesting a reaction-limited aggregation (RLA) process [17-19]. A rapid increase of $I(q)$ over time during the first $160 \mathrm{~s}$ [see Fig. S2(a) in the Supplemental Material [15] ] of the measurements indicates a structural evolution $[7,20,21]$. During this step, major protein components unfold and assume a different conformational state, which facilitates an enhanced exposure of the hydrophobic and sulfhydryl (SH) groups [7,20]. This, in turn, reduces $\alpha$ helix structures and increases $\beta$-sheet components [7,22]. The rate of increase in intensity in the USAXS profiles reduces with time [see Figs. S2(b) and S2(c) in the Supplemental Material [15]] and eventually stabilizes, showing no further structural change within the measured $q$ range. The stabilization of $I(q)$ suggests that the major structural evolution corresponding to the denaturation and formation of the gel network is completed over the measured length scales ( 60-2000 nm).

Next, we calculate the exponent $\delta$ of the power law relation of $I(q)$ as a function of $q\left(I(q) \sim q^{-\delta}\right)$ [23] in the low $q$ regime $\left(0.0046-0.0077 \mathrm{~nm}^{-1}\right)$ to determine a characteristic parameter, the fractal dimension, which indicates the extent of filling of the available space by the structure in question [6,24-26]. $\delta$ as a function of $t_{w}$ is shown in Fig. 1(b). $\delta$ gradually increases with $t_{w}$ and eventually saturates at a value of $\approx 2$ confirming that the present aggregation can be described by the RLA process [19].

We further characterize the development of the characteristic length scale $\xi$ of the gel network and determine the mesh size of the gel in the final stages using the OrnsteinZernike equation (see the Supplemental Material [15]) $[27,28]$ and shown in Fig. 1(c). The mesh size of the network $\xi$ (after $t_{w} \sim 300 \mathrm{~s}$ ) asymptotically approaches a value of ca. $400 \mathrm{~nm}$. Note that within a few days of age of the egg, the sample freshness does not alter our result significantly as suggested by the SAXS measurements (see Fig. S4 in the Supplemental Material [15]). We then investigated the dynamics of the gel at the length scales related to such mesh size aiming to understand its relation with the structural evolution.

The evolution of dynamics is obtained from the two-time correlation (TTC) defined as the covariance of the scattered intensity $[12,13,29-35]$

$$
C_{I}\left(q, t_{1}, t_{2}\right)=\frac{\left\langle I_{p}\left(q, t_{1}\right) I_{p}\left(q, t_{2}\right)\right\rangle_{\text {pixels }}}{\left\langle I_{p}\left(q, t_{1}\right)\right\rangle_{\text {pixels }}\left\langle I_{p}\left(q, t_{2}\right)\right\rangle_{\text {pixels }}},
$$

where $I_{p}$ is the intensity at the pixel $p$, and $\langle. .\rangle_{\text {pixels }}$ indicates an average over the pixels within a given wave vector range $q \pm \delta q . t_{1}$ and $t_{2}$ are the times at which the intensity was measured, $t_{w}=\left(t_{1}+t_{2}\right) / 2$ is the distance along the diagonal $\left(t_{1}=t_{2}\right)$, and the time difference $t=\left|t_{1}-t_{2}\right|$ is the distance away from the diagonal (see Fig. S5 in the Supplemental Material [15]) [12,13, 29-37]. During the gelation, we performed five consecutive XPCS runs (each run corresponding to $160 \mathrm{~s}$ ) at different fresh sample spots, and the corresponding TTC are depicted in Figs. 2(a)-2(e) to follow the evolution throughout the entire measurement time.

In Figs. 2(a)-2(c), a pronounced slowing-down of the dynamics with $t_{w}$ is observed. Note that the dynamics is observable only after $160 \mathrm{~s}$ [starting from Fig. 2(b)] when the major part of the structural evolution has taken place [see Figs. 1(a)-1(c)]. The inset in Fig. 2 schematically

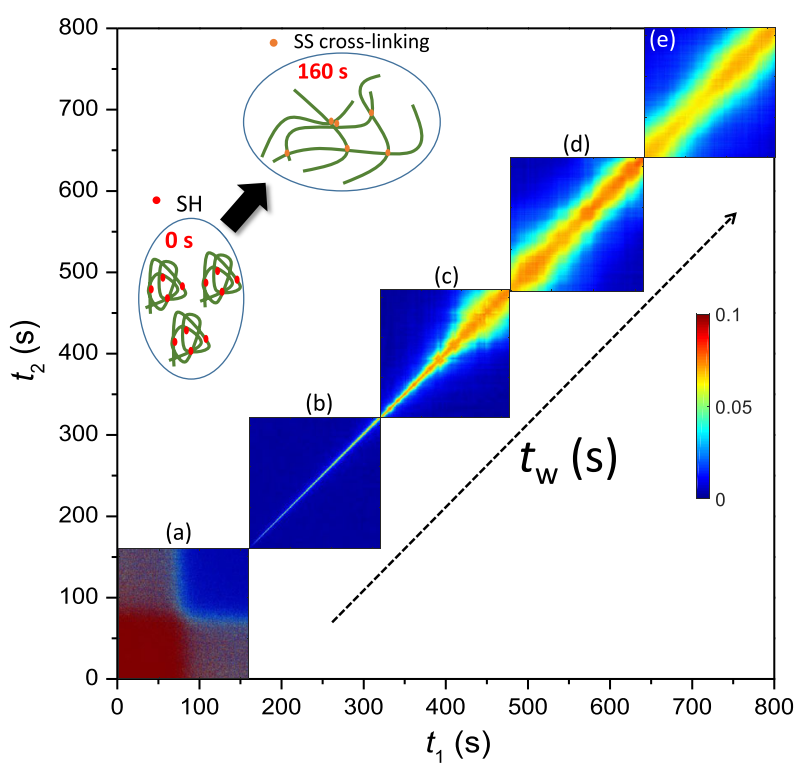

FIG. 2. TTC $\left(C_{I}-1\right)$ collected at $80^{\circ} \mathrm{C}\left(\right.$ at $\left.q=0.01 \mathrm{~nm}^{-1}\right)$ in the time interval of (a) 0-160 s, (b) 160-320 s, (c) 320-480 s, (d) 480-640 s, and (e) 640-800 s after reaching the temperature. Inset schematic shows the native state of the proteins before denaturation $\left(0 \mathrm{~s}\right.$ after heating at $\left.80^{\circ} \mathrm{C}\right)$ and after unfolding due to heat denaturation $\left(160 \mathrm{~s}\right.$ after heating at $\left.80^{\circ} \mathrm{C}\right)$. 
illustrates the native state of the proteins in the beginning of the experiment (at $0 \mathrm{~s}$ ) and the unfolded cross-linked state after $160 \mathrm{~s}$ of heating that eventually forms a network [38]. At this stage, the $\mathrm{SH}$ groups are exposed to the exterior of the protein molecules and are able to interact within the molecules as well as with the neighboring molecules, which leads to disulfide link formation [7].

The observed growth of the dynamics is suggestive of an aging of the gel $[39,40]$. At a later stage [Figs. 2(d) and 2(e)], the relaxation time stabilizes around a mean value, and interestingly, shows a periodic temporal fluctuation, which is indicative of dynamical heterogeneity.

The relaxation times extracted from the fit of $g_{2}(q, t)$ (see Fig. S6 in the Supplemental Material [15]) as a function of $t_{w}$ are shown in Fig. 3. As we observe in the TTC, the growth of $\tau$ starts when the rapid change in structure (in the measured $q$ range) ends. This observation suggests that the microscopic dynamics during the gelation process (approximately the first $160 \mathrm{~s}$ after the heating started) is too fast to be captured within our experimental time window. Thus, the measured dynamics after $160 \mathrm{~s}$ of heating, when there are no significant structural changes, could be related to the aging or the stabilization of the networks through local reorganization that occurs in a gel (after the gel formation). The initial rapid increase in $\tau$ [the orange background in Fig. 3(a)] follows an exponential growth as a function of $t_{w}$ [shown by the dashed line in Fig. 3(a)]. This behavior is visible for all the measured $q$ values [see Fig. 3(a)]. After $\sim 500 \mathrm{~s}$, the dynamics stabilizes [the green background in Fig. 3(a)] at a characteristic time fluctuating around a mean value of a few tens of seconds [ca. $\tau<100 \mathrm{~s}$; see the inset of Fig. 3(a)]. This observation seems to be unique for the

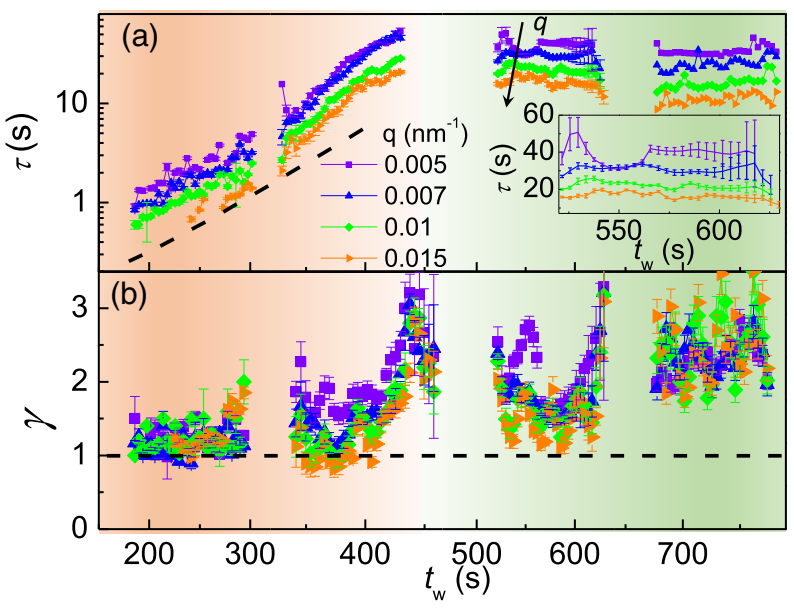

FIG. 3. (a) Evolution of relaxation time as a function of $t_{w}$ at $80{ }^{\circ} \mathrm{C}$ at different $q$ as indicated in the legend (in $\mathrm{nm}^{-1}$ ). The inset shows the data within the time window $t_{w}=480-640 \mathrm{~s}$ on a linear scale to visualize the temporal fluctuations. The dashed black line represents an exponential growth function. (b) The exponent $\gamma$ as a function of $t_{w}$ shows a transition from an exponential to a compressed exponential decay. present complex system distinguishing it from conventional colloidal or polymeric gels [41-43].

The stretching exponent $\gamma$ displays a pronounced dependence on the waiting time evidencing the evolution of the dynamics during gelation. In the early times, we observe an exponential $(\gamma \sim 1)$ correlation function. Beginning at $\sim 300$ s the values of $\gamma$ start to increase, reaching values of more than 2 [in Fig. 3(b)] and then fluctuate well above 1 indicating a transition from an exponential to a steeper-thanexponential dynamics $[32,43]$.

More information about the dynamics can be obtained from the $q$ dependence of $\gamma$ [see Figs. S8(a)-S8(d) in the Supplemental Material [15]] and $\tau(q)$ [see Fig. S8(e) in the Supplemental Material [15]]. We observe a strong $q$ dependence of $\gamma$ at intermediate waiting times ( 320-640 s). Interestingly, $\gamma$ shows weak or no $q$ dependence in the initial waiting times (before $300 \mathrm{~s}$ ) as well as at the later stages (after 600 s). Figure S8(e) in the Supplemental Material [15] depicts the $q$ dependence of $\tau(q)$ modeled with a power law $\left[\tau(q) \sim q^{-\alpha}\right]$. The data indicate $\alpha \approx 1$ throughout the measurement time, ruling out Brownian diffusive motion $(\alpha=2)$ of the aggregates. Instead, the data suggest that the particle displacement increases linearly with time, which, in combination with a compressed exponential correlation function $(\gamma>1)$, are the signatures of intermittent hyperdiffusive dynamics progressing by a limited number of single decorrelation events $[39,41,44,45]$.

The $q$ dependence of $\gamma$ can be understood in the framework of the relaxation of stresses generated during the network formation, i.e., during the random crosslinking. At the early stages, during the establishment of the gel network through cross-linking, the dynamics is nearly exponential [see Figs. 3(a) and 3(b)] [46]. As the sample ages, the increased cross-linking accumulates the stresses in the gel. The relaxation of such trapped stresses through large-scale rearrangements leads to the compressed exponential relaxation $[43,46]$. We speculate that, at these intermediate stages, the possibility of the inhomogeneous distribution of the stress in space in the cross-linked network leads to the $q$-dependent $\gamma$. As the sample ages further, such inhomogeneity reduces, and thus, the dynamics exhibits $\gamma \approx 2$ for the entire $q$ range.

We further explore the gel dynamics by calculating the average spatial extension $\sigma$ of the decorrelation events adopting the method described in Refs. [47-50]. Applying this methodology, the intermediate scattering function $f(t)=\sqrt{\left[g_{2}(t)-1\right] / \beta}$ is expressed as a series of decorrelation events of the gel network

$$
f(t)=\sum_{N=0}^{\infty} P_{t}(N) h(N)
$$

$P_{t}(N)$ is the probability of $N$ events occurring during a time interval $t$ given by a Poisson distribution 

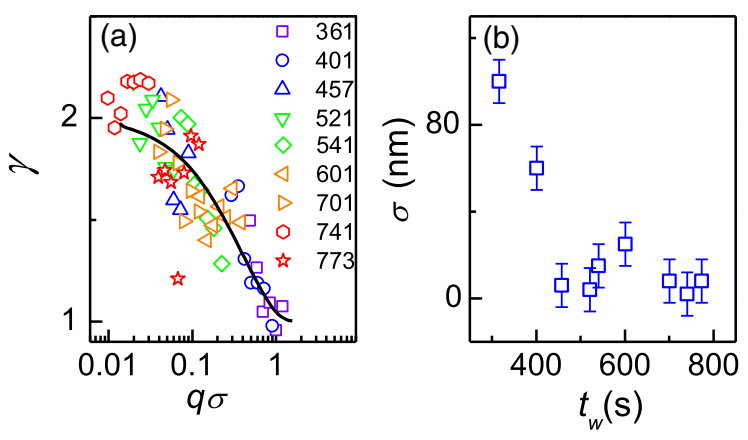

FIG. 4. (a) $\gamma$ as a function of $q \sigma$ at different $t_{w}$ (s) as indicated in the legend. The black solid line represents the theoretical curve used to obtain the values of $\sigma$. (b) $\sigma$ as a function of $t_{w}$ showing a reduction with waiting time.

$\left[=\left(\Gamma_{0} t\right)^{N} \exp \left(-\Gamma_{0} t\right) / N !, 1 / \Gamma_{0}\right.$ being the mean time between events], and $h(N)$ is the decorrelation rate of $f(t)$ after $N$ events of size $\sigma$ given by

$$
h(q, N) \simeq \exp \left[-(q N \sigma)^{2}\right]
$$

for a ballistic motion (i.e., $\alpha=1$ ) [50]. $\Gamma_{0}$ is kept constant at 0.1 and $N$ is varied from 0 to 100 . With this, we obtain theoretical $g_{2}$ functions yielding values of $\gamma$ as a function of $q \sigma$.

The black line in Fig. 4(a) shows $\gamma$ as a function of $q \sigma$ extracted from the modeled $g_{2}$ functions. The data superimpose with the black line when the corresponding $q$ values of the datasets are multiplied by individual values of $\sigma\left(t_{w}\right)$ for each time interval. Figure 4(b) shows $\sigma\left(t_{w}\right)$ as a function of $t_{w}$ indicating a gradual reduction in $\sigma$ with increasing $t_{w}$. Larger values of $\sigma$ at earlier times imply that the relaxation of the system, immediately after gel formation, progresses through a small number of relatively large rearrangement events. At this stage, the relatively soft and flexible structure of the gel network facilitates such large restructuring events. With increasing $t_{w}$, the network stiffens gradually as indicated by the increase in elastic modulus with time in a colloidal gel [51], despite the absence of further structural changes observed in Fig. 1. As a result of this stiffening, the system relaxes slowly through a larger number of small decorrelation events, which leads to a reduced $\sigma$ and an increased $\tau$.

We observed in Figs. 2(d) and 2(e) that $\tau$ exhibits a temporal modulation. Such fluctuations could occur due to spatial correlations of the dynamics that restrict the number of independent regions in the system and result in discrete rearrangement events in the gel. These fluctuations, thus, indicate the presence of dynamical heterogeneity in the system $[12,52]$. We quantified the dynamical heterogeneity by calculating the fourth order correlation function or the dynamic susceptibility given by $[12,13,29,39]$
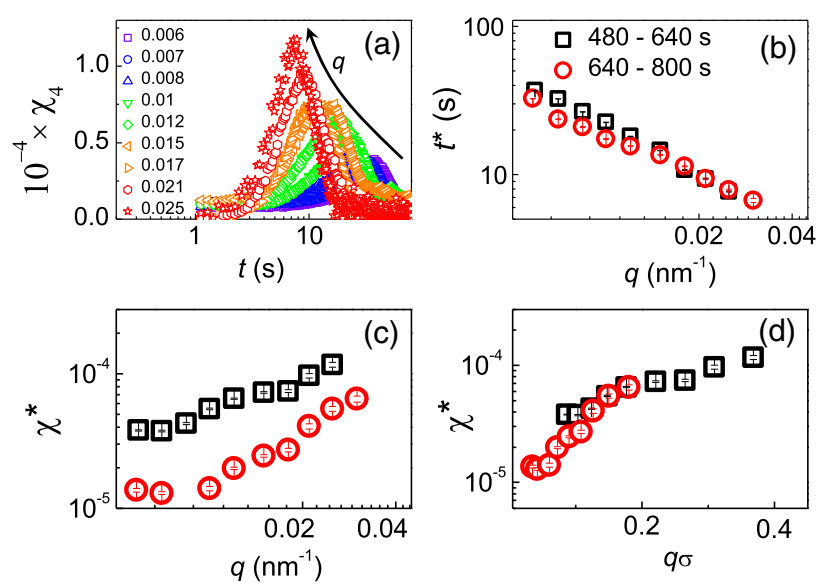

FIG. 5. (a) $\chi_{4}$ evaluated from the TTC [shown in Fig. 2(d)] as a function of delay time, (b) $t^{\star}$ as a function of $q$ for two different waiting times showing no significant change with age, and (c) $\chi^{\star}$ as a function of $q$ showing a monotonic $q$ dependence. Error bars are smaller than the symbols. (d) $\chi^{\star}$ as a function of $q \sigma$ showing that the degree of heterogeneity as a function of $t_{w}$ falls on a single curve.

$$
\chi_{4}=\frac{\left\langle C_{I}^{2}\left(q, t_{1}, t\right)\right\rangle_{t_{1}}-\left\langle C_{I}\left(q, t_{1}, t\right)\right\rangle_{t_{1}}^{2}}{\left\langle C_{I}\left(q, t_{1}, t=0\right)\right\rangle_{t_{1}}^{2}} .
$$

Figure 5(a) shows the calculated $\chi_{4}$ as a function of the delay time. The peak position $t^{\star}$ of $\chi_{4}$ corresponds to the characteristic time of the heterogeneous dynamics, while its height $\chi^{\star}$ gives a measure of the degree of heterogeneity in the dynamics. The extracted $\chi^{\star}$ and $t^{\star}$ are summarized as a function of $q$ in Figs. 5(b) and 5(c). Note that the absolute values of $t^{\star}$ do not change significantly with $t_{w}$. Nevertheless, the peak height $\chi^{\star}$ significantly reduces with $t_{w}$. It is thus indicating that the degree of heterogeneity reduces with $t_{w}[41,48]$, possibly through structural rearrangements as the elementary steps along the direction of its equilibrium state. This is in agreement with our observation that the size $\sigma$ of the restructuring events decreases with $t_{w}$ [Fig. 4(b)]. Consequently, the curves of $\chi^{\star}$ as a function of $q \sigma$ at different $t_{w}$ fall on the same curve [Fig. 5(d)].

The length scale dependence of $\chi_{4}$ reveals that $t^{\star}$ decreases with increasing $q$ as $t^{\star} \sim 1 / q$ [Fig. 5(b)] [39] and $\chi^{\star}$ increases with $q$ [Fig. 5(c)] [53]. The observation of $\chi^{\star}$ increasing with $q$ has also been observed previously in attractive colloidal fractal gels [48]. In such type of gels, the volume fractions can be very small compared to any noninteracting colloidal gel $[32,54]$. The gelation of the whole egg white where the total volume fraction of proteins is rather low also supports the possibility of such type of dilute colloidal fractal-like gel formation. The gelation of proteins is favored when the electrostatic repulsion between the protein molecules is substantially reduced [55]. Our results thus indicate that the electrostatic repulsive forces between the proteins present in their native states in an egg 
white are reduced after heat-induced denaturation. Eventually, the system exhibits an interesting attractive colloidal gel-like dynamics. The observed ballistic dynamics $\left(\tau \sim q^{-1}\right)$ with $\chi^{\star} \sim q$ is possibly due to slow compaction of the gel through discrete rearrangements [48]. At a smaller length scales, especially at the length scales of the mesh size $(\sim 400 \mathrm{~nm}$ and corresponding $q=0.016 \mathrm{~nm}^{-1}$ ), these events might be distinct and observable, which results in higher $\chi^{\star}$ values at smaller length scales (i.e., at higher $q$ ). On the other hand, while probing at large length scales (i.e., at smaller $q$ ), the system appears to be rather homogeneous with less temporal fluctuation in the dynamics (i.e., smaller $\chi^{\star}$ values).

To conclude, we simultaneously investigate the kinetics of thermal gelation and dynamics of an egg white gel by employing state-of-the-art XPCS along with USAXS on length scales of the network mesh size. A rapid increase in scattering intensity at the onset of the reaction-limited aggregation process is observed. The microscopic dynamics reveals an exponential rise of the relaxation time and a subsequent steady-state ballistic and heterogeneous dynamics. We discuss the observed dynamics in the framework of a stress-activated ballistic dynamics in a low volume fraction attractive colloidal gel. Our study paves the way for future experiments following protein gelation and aggregation on length scales from $\mathrm{nm}$ to microns shedding light on processes highly relevant for the food industry and soft matter physics.

The authors would like to thank the DESY (Hamburg, Germany) for beam time. The authors also thank Motiur Rahman Khan for his suggestions. This work was supported by the DFG and BMBF. N. B. acknowledges the Alexander von Humboldt-Stiftung for a postdoctoral research fellowship, A. R. acknowledges support from the Studienstiftung des deutschen Volkes and C. G. acknowledges BMBF (Grants No. 05K19PS1 and No. 05K20PSA) for financial support.

*nafisa.begam@uni-tuebingen.de

†fajun.zhang@uni-tuebingen.de

*christian.gutt@uni-siegen.de

§frank.schreiber@uni-tuebingen.de

[1] V. Raikos, L. Campbell, and S. R. Euston, Food Hydrocolloids 21, 237 (2007).

[2] M. Fach, L. Radi, and P. R. Wich, J. Am. Chem. Soc. 138, 14820 (2016).

[3] N. Koshoubu, H. Kanaya, K. Hara, S. Taki, E. Takushi, and K. Matsushige, J. Appl. Phys. 32, 4038 (1993).

[4] P. W. Gossett, S. Rizvi, and R. C. Baker, Food Technol. 38, 67 (1984).

[5] T. Nojima and T. Iyoda, NPG Asia Mater. 10, e460 (2018).

[6] S. Ikeda, E. A. Foegeding, and T. Hagiwara, Langmuir 15, 8584 (1999).

[7] Y. Mine, T. Noutomi, and N. Haga, J. Agric. Food Chem. 38, 2122 (1990).
[8] T. M. Blois, H. Hong, T. H. Kim, and J. U. Bowie, J. Am. Chem. Soc. 131, 13914 (2009).

[9] J. Li, Y. Zhang, Q. Fan, C. Teng, W. Xie, Y. Shi, Y. Su, and Y. Yang, Food Chem. 250, 1 (2018).

[10] M. Hennig, F. Roosen-Runge, F. Zhang, S. Zorn, M. W. A. Skoda, R. M. J. Jacobs, T. Seydel, and F. Schreiber, Soft Matter 8, 1628 (2012).

[11] M. Katava, G. Stirnemann, M. Zanatta, S. Capaccioli, M. Pachetti, K. L. Ngai, F. Sterpone, and A. Paciaroni, Proc. Natl. Acad. Sci. U.S.A. 114, 9361 (2017).

[12] H. Conrad, F. Lehmkühler, B. Fischer, F. Westermeier, M. A. Schroer, Y. Chushkin, C. Gutt, M. Sprung, and G. Grübel, Phys. Rev. E 91, 042309 (2015).

[13] S. K. Sinha, Z. Jiang, and L. B. Lurio, Adv. Mater. 26, 7764 (2014).

[14] A. Fluerasu, P. Kwasniewski, C. Caronna, F. Destremaut, J.-B. Salmon, and A. Madsen, New J. Phys. 12, 035023 (2010).

[15] See Supplemental Material at http://link.aps.org/ supplemental/10.1103/PhysRevLett.126.098001 for additional details on experimental setup and analysis.

[16] S. Da Vela, N. Begam, D. Dyachok, R. S. Schäufele, O. Matsarskaia, M. K. Braun, A. Girelli, A. Ragulskaya, A. Mariani, F. Zhang, and F Schreiber, J. Phys. Chem. Lett. 11, 7273 (2020).

[17] F. Zhang, D. Dressen, M. Skoda, R. Jacobs, S. Zorn, R. Martin, C. Martin, G. Clark, and F. Schreiber, Eur. Biophys. J. 37, 551 (2008).

[18] A. Zaccone, D. Gentili, H. Wu, and M. Morbidelli, J. Chem. Phys. 132, 134903 (2010).

[19] S. Jungblut, J.-O. Joswig, and A. Eychmüller, Phys. Chem. Chem. Phys. 21, 5723 (2019).

[20] A. Halabi, A. Deglaire, P. Hamon, S. Bouhallab, D. Dupont, and T. Croguennec, Food Chem. 302, 125296 (2020).

[21] A. Kato and T. Takagi, J. Agric. Food Chem. 36, 1156 (1988).

[22] H. Hofmann, U. Weininger, C. Low, R. P. Golbik, J. Balbach, and R. Ulbrich-Hofmann, J. Am. Chem. Soc. 131, 140 (2009).

[23] C. Schmitt, C. Moitzi, C. Bovay, M. Rouvet, L. Bovetto, L. Donato, M. E. Leser, P. Schurtenberger, and A. Stradner, Soft Matter 6, 4876 (2010).

[24] D. Johansen, J. Trewhella, and D. P. Goldenberg, Protein Sci. 20, 1955 (2011).

[25] A. Zaccone, J. J. Crassous, and M. Ballauff, J. Chem. Phys. 138, 104908 (2013).

[26] C. Arzeni, O. E. Perez, and A. M. R. Pilosof, Food Hydrocolloids 29, 308 (2012).

[27] C. C. Han and A.Z. Akcasu, Scattering and Dynamics of Polymers: Seeking Order in Disordered Systems (John Wiley \& Sons, New York, 2011).

[28] D. Jia and M. Muthukumar, Macromolecules 53, 90 (2020).

[29] A. Madsen, R. L. Leheny, H. Guo, M. Sprung, and O. Czakkel, New J. Phys. 12, 055001 (2010).

[30] Z. Evenson, B. Ruta, S. Hechler, M. Stolpe, E. Pineda, I. Gallino, and R. Busch, Phys. Rev. Lett. 115, 175701 (2015).

[31] D. Van't Zand, Y. Chushkin, L. Belkoura, C. Lobo, R. Strey, K. Lyakhova, and P. Clegg, Soft Matter 8, 4062 (2012).

[32] A. Fluerasu, A. Moussaïd, A. Madsen, and A. Schofield, Phys. Rev. E 76, 010401(R) (2007). 
[33] F. Perakis and C. Gutt, Phys. Chem. Chem. Phys. 22, 19443 (2020).

[34] G. Grübel and F. Zontone, J. Alloys Compd. 362, 3 (2004).

[35] V. Nigro, B. Ruzicka, B. Ruta, F. Zontone, M. Bertoldo, E. Buratti, and R. Angelini, Macromolecules 53, 1596 (2020).

[36] A. Fluerasu, A. Moussaïd, P. Falus, H. Gleyzolle, and A. Madsen, J. Synchrotron Radiat. 15, 378 (2008).

[37] K. Yu, T. Xie, J. Leng, Y. Ding, and H. J. Qi, Soft Matter 8, 5687 (2012).

[38] J.-W. Chang, C.-G. Wang, C.-Y. Huang, T.-D. Tsai, T.-F. Guo, and T.-C. Wen, Adv. Mater. 23, 4077 (2011).

[39] V. Trappe, E. Pitard, L. Ramos, A. Robert, H. Bissig, and L. Cipelletti, Phys. Rev. E 76, 051404 (2007).

[40] J. H. Cho, R. Cerbino, and I. Bischofberger, Phys. Rev. Lett. 124, 088005 (2020).

[41] H. Guo, S. Ramakrishnan, J. L. Harden, and R. L. Leheny, J. Chem. Phys. 135, 154903 (2011).

[42] R. Bandyopadhyay, D. Liang, J. L. Harden, and R. L. Leheny, Solid State Commun. 139, 589 (2006).

[43] M. Bouzid, J. Colombo, L. V. Barbosa, and E. Del Gado, Nat. Commun. 8, 15846 (2017).

[44] L. Ramos and L. Cipelletti, Phys. Rev. Lett. 87, 245503 (2001).

[45] M. Bellour, A. Knaebel, J. L. Harden, F. Lequeux, and J.-P. Munch, Phys. Rev. E 67, 031405 (2003).
[46] H. Guo, G. Bourret, M. K. Corbierre, S. Rucareanu, R. B. Lennox, K. Laaziri, L. Piche, M. Sutton, J. L. Harden, and R. L. Leheny, Phys. Rev. Lett. 102, 075702 (2009).

[47] L. Cipelletti, L. Ramos, S. Manley, E. Pitard, D. A. Weitz, E. E. Pashkovski, and M. Johansson, Faraday Discuss. 123, 237 (2003).

[48] A. Duri and L. Cipelletti, Europhys. Lett. 76, 972 (2006).

[49] D. Aarts, R. Dullens, and H. Lekkerkerker, New J. Phys. 7, 40 (2005).

[50] C. Caronna, Y. Chushkin, A. Madsen, and A. Cupane, Phys. Rev. Lett. 100, 055702 (2008).

[51] S. Manley, B. Davidovitch, N. R. Davies, L. Cipelletti, A. E. Bailey, R. J. Christianson, U. Gasser, V. Prasad, P. N. Segre, M. P. Doherty et al., Phys. Rev. Lett. 95, 048302 (2005).

[52] A. M. Puertas, M. Fuchs, and M. E. Cates, J. Phys. Chem. B 109, 6666 (2005).

[53] P. Mayer, H. Bissig, L. Berthier, L. Cipelletti, J. P. Garrahan, P. Sollich, and V. Trappe, Phys. Rev. Lett. 93, 115701 (2004).

[54] N. Khalil, A. de Candia, A. Fierro, M. P. Ciamarra, and A. Coniglio, Soft Matter 10, 4800 (2014).

[55] L. Campbell, V. Raikos, and S. R. Euston, Food/Nahrung 47, 369 (2003). 